\title{
Acrocarpic mosses (Bryophyta) of Chapada das Mesas National Park, Maranhão, Brazil
}

\author{
Regigláucia Rodrigues de Oliveira ${ }^{1}$, Hermeson Cassiano de Oliveira ${ }^{1,2}$, Denilson Fernandes \\ Peralta $^{3}$, Gonçalo Mendes da Conceição ${ }^{1}$ \\ 1 Programa de Pós-Graduação em Biodiversidade, Ambiente e Saúde, Universidade Estadual do Maranhão, Praça Duque de Caxias, Morro do \\ Alecrim, s/n, 65055-310, Caxias, Maranhão, Brazil. 2 Universidade Estadual do Piauí, Campus Heróis do Jenipapo, Av. Sto. Antônio, s/n, 64280- \\ 000, Campo Maior, Piauí, Brazil. 3 Instituto de Botânica de São Paulo, Av. Miguel Stéfano, 3687, 04301-012 - São Paulo, São Paulo, Brazil. \\ Corresponding author: Regigláucia Rodrigues de Oliveira, regiglaucia@hotmail.com
}

\begin{abstract}
Chapada das Mesas National Park is located in southwestern Maranhão state, Brazil and comprises several forest types characteristic of the Brazilian Cerrado. We contribute to the knowledge of the distribution of Brazilian bryoflora and provide a list of moss species of Chapada das Mesas National Park. A total of 38 species of acrocarpic mosses were found, with Fissidentaceae being the richest family with 11 species. Of the species recorded, 22 are new occurrences for the state of Maranhão, and 4 of these are new records for the Northeast Region of Brazil. Most of the species recorded are broadly distributed in Brazil (66\%), while restricted species represented just $10 \%$. The most common acrocarpic moss species were Hyophila involuta (Hook.) A. Jaeger and Octoblepharum albidum Hedw.
\end{abstract}

\section{Key words}

Bryophytes; Cerrado; rupestrian fields.

\section{Introduction}

Chapada das Mesas National Park (CMNP) is located in the southwest region of the state of Maranhão, Brazil. It was created by presidential decree on December 2005, as a result of a historical process of community organization in the region, for the establishment of a Conservation Unit to protect the region of the Chapadas das Mesas against the advance of the agricultural frontier and other projects that could endanger its ecological value and compromise its ecotourism potential (Moraes and Lima 2007). The various vegetation types present in CMNP are
Cerrado (sensu strict) and campo sujo (savanna), along with forest formations of cerradão (seasonal forest), mata seca (dry forest) and the so-called marginal forests comprising matas de galeria (gallery forests) and buritizais (palm swamps) (Moraes and Lima 2007). There are also numerous water sources in the form of 3 important river basins: Parnaíba, Araguaia/Tocantins, and São Francisco.

According to Galinkin et al. (2004), the southwestern region of Maranhão is important for the maintenance of Brazilian biodiversity because it encompasses an ecotone between 3 biomes, the Cerrado, Amazon, and Caatinga. Thus, it represents an area with high potential 


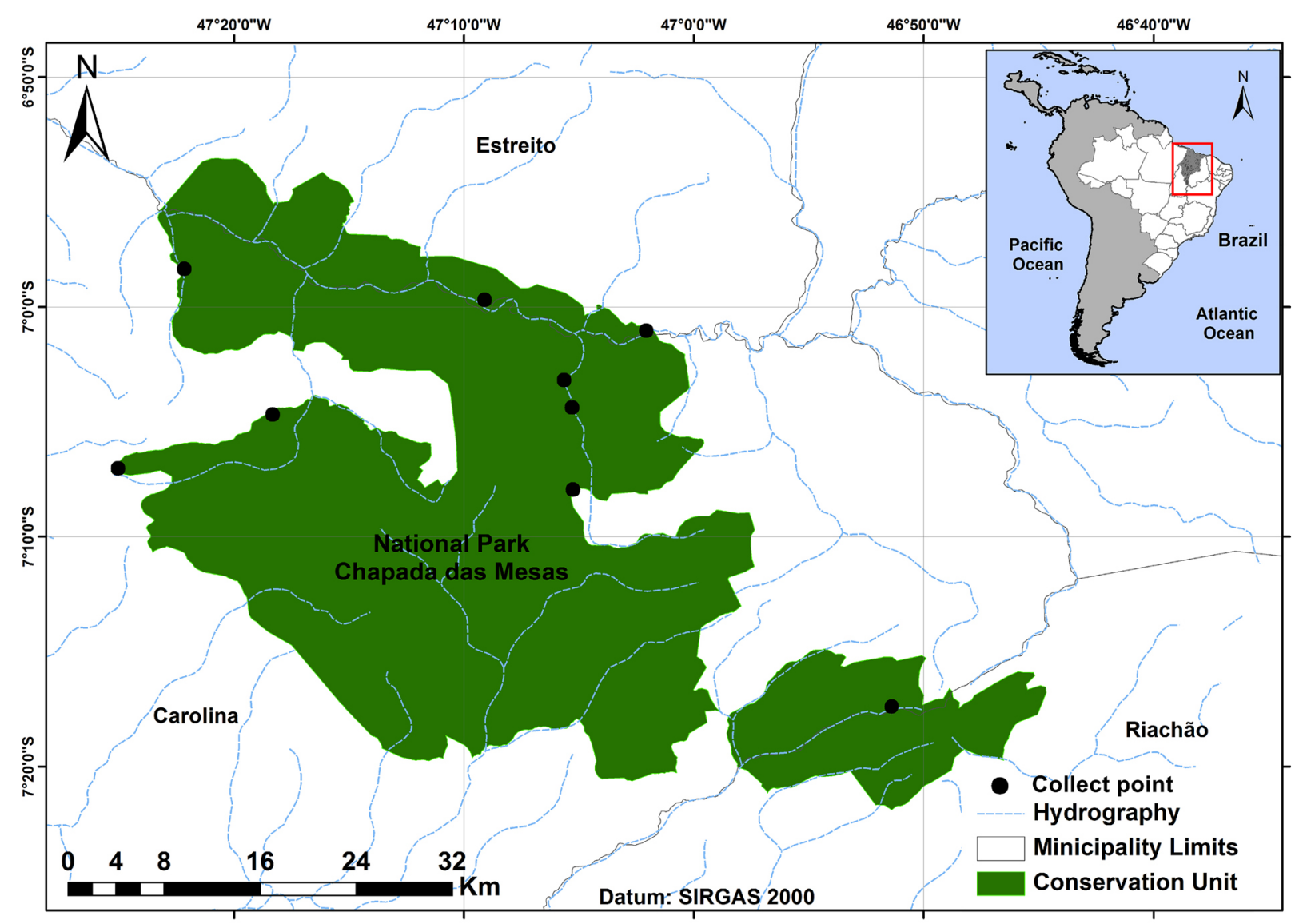

Figure 1. Geographic location of CMNP in Brazil and the state of Maranhão.

for sheltering high levels of richness and abundance of flora and fauna and serves as significant component of the Araguaia-Bananal ecological corridor. Although the Cerrado is recognized as one of the main centers of diversity and endemism of plant species in Brazil (Medeiros 2011), there is little information about the bryoflora of the Cerrado of Maranhão and in particular of CMNP. The destruction of the Cerrado has accelerated as this biome is being replaced by monocultures. In view this, the need to carry out inventories of the bryoflora has become extremely relevant.

Sixty species of mosses are known from the state of Maranhão (Costa and Peralta 2015). However, inventories of bryophytes around waterfalls have yet to be undertaken in the state even though a survey in Serra da Canastra National Park revealed rich moss flora in similar habitats (Carmo and Peralta 2016).

The acrocarpic growth form is evidenced in mosses that present a perichaetium produced at the apex of a main branch (La Farge-England1996). The high diversity of acrocarpic mosses in the state of Maranhão is remarkable, as shown in publications on the bryoflora of the state (Peralta et al. 2011, Vieira et al. 2017).

The great importance of CMNP for the preservation of endemic species and its maintenance as a Conservation Unit make it an interesting area for study. Therefore, we made a floristic inventory of the acrocarpic mosses of CMNP with our goal to increase knowledge about the geographic distribution of bryophytes in Brazil.

\section{Methods}

Study area. CMNP has an area of 161,000 ha divided between 2 administrative areas. The larger area (140,000 ha) covers parts of the municipalities of Carolina and Estreito, while the smaller (19,000 ha) covers the part of municipality of Riachão. The relief is characterized by the presence of sandstone hills with elevations between $250 \mathrm{~m}$ and $524 \mathrm{~m}$ (Fig. 1).

According to the Köppen climate classification, the climate of the region is Tropical Humid, with high temperatures throughout the year. There are 2 well-defined seasons: a dry summer from May to October and a rainy winter from November to April. The average annual mean temperature is $26.1^{\circ} \mathrm{C}$, with a minimum temperature of $25.2{ }^{\circ} \mathrm{C}$ in January to September and a maximum of $36^{\circ} \mathrm{C}$ in July and August. Annual rainfall ranges from 1,250 to $1,500 \mathrm{~mm}$ per year (Lima et al. 2007).

The CMNP is almost entirely on yellow latosol soil with a characteristic deep profile, along with the constant presence of sedimentary sands, which are characteristic of these tabular reliefs. The main types of soils found in the region of CMNP are quartz sands, cambisols, and redyellow latosols (Martins et al. 2017).

There are numerous springs within CMNP which supply 3 important watersheds: Parnaíba, Araguaia/Tocantins, and São Francisco. The Farinha River, one of the larger rivers of CMNP, is a tributary of the south bank of the Tocantins River, which is a very important water source 
for the entire central region of the country (Marques and Amorim 2014).

Sampling, herborization and identification. Authorization for the collection and transport of specimens was approved by the Instituto Chico Mendes de Conservação da Biodiversidade (ICMBio number 50981).

Our collection expeditions were carried out during both the dry and rainy seasons and were focussed on areas near waterfalls, but also covered other areas and substrates. We gave special attention to the habitats most favorable for the colonization by bryophytes, such as waterfalls and the vegetation along stream banks. We collected and herborized the samples according to Frahm (2003), with the specimens being collected manually or with a knife and/or spatula and placed in brown paper bags. Data on substrate colonization follow the bryocenological groups of Robbins (1952), and included corticicolous (Co), epixilous (Epx), epiphyllous (Epf), rupicolous $(\mathrm{Ru})$, and terricolous $(\mathrm{Te})$.

We identified the samples and incorporated them into the Herbarium Prof. Aluízio Bittencourt (HABIT), Universidade Estadual do Maranhão (UEMA), Centro de Estudos Superiores de Caxias (CESC), with duplicates incorporated into the Herbarium "Maria Eneyda Pacheco Kauffman Fidalgo" (SP) at the Instituto de Botânica de São Paulo.

Species identification was accomplished in consultation with Frahm (1991), Zander (1993), Sharp et al. (1994), Buck (1998), Gradstein et al. (2001), Câmara (2008), Yano (2011), Yano and Peralta (2011), Valente et al. (2011), Bordin and Yano (2013), and Luizi-Ponzo et al. (2013).

The classification system adopted is that of Goffinet et al. (2009). Brazilian geographical distributions were categorized according to Valente and Pôrto (2006), who considered bryophytes that occur in 1 to 4 Brazilian states as "restricted"; those occurring in 5 to 9 states as "moderately distributed"; and those occurring in 10 or more Brazilian states as "broadly distributed". The geographical distributions follow Forzza et al. (2010), Costa et al. (2011), Costa and Peralta (2015) and Carmo and Peralta (2016).

\section{Results}

We found 38 species of acrocarpic mosses representing 18 genera and 7 families (Table 1). The richest bryophyte family in CMNP was Fissidentaceae (11 spp.), followed by Calymperaceae ( 9 spp.), Pottiaceae (7 spp.), Dicranaceae, Bryaceae and Leucobryaceae (3 spp. each), and Bartramiaceae (2 spp.) (Fig. 2). The most common species were Hyophila involuta (Hook.) A. Jaeger and Octoblepharum albidum Hedw., each of which occurred in 42 samples. Most of the species recorded are broadly distributed in Brazil (66\%), while restricted species represented just 10\% (Fig. 3).

Of species found, $22(55 \%)$ are new occurrences

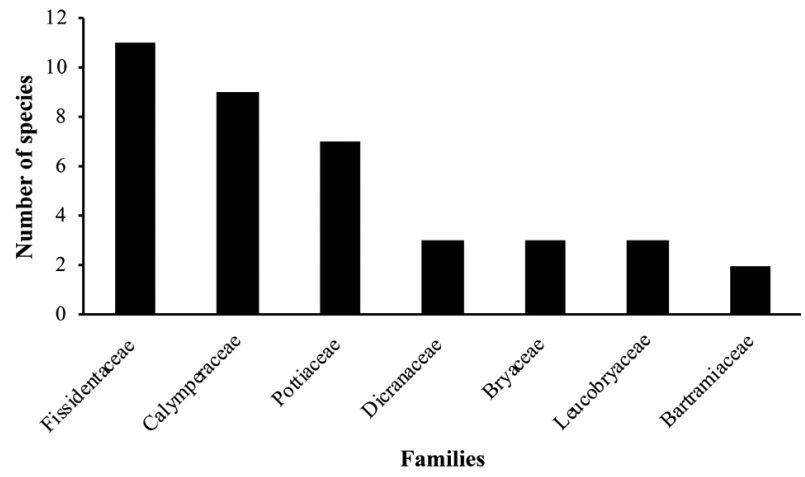

Figure 2. Richness of acrocarpic moss families in CMNP, Maranhão, Brazil.

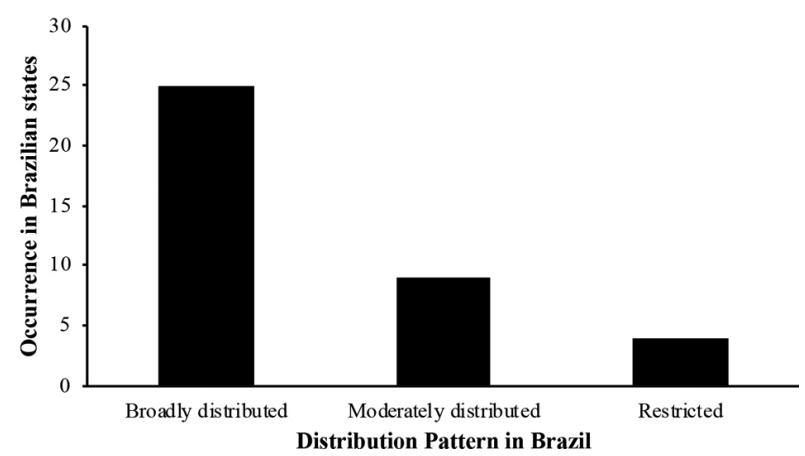

Figure 3. Distribution pattern of species in Brazilian states.

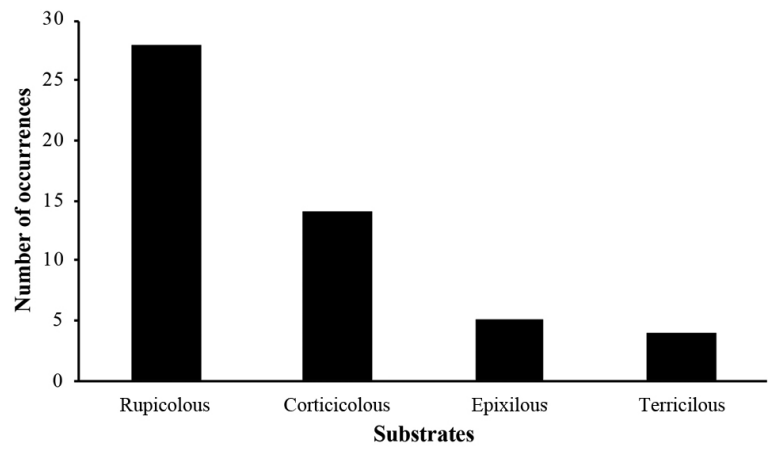

Figure 4. Percentage of substrates colonized by acrocarpic moss species occurring in CMNP, Maranhão, Brazil.

for the state of Maranhão, of which, Leucobryum laevifolium Broth., Syrrhopodon tortilis Hampe, Fissidens steerei Grout., and Tortella tortuosa (Schrad. ex Hedw.) Limpr. constitute new records for the Northeast Region of Brazil, and represented $8 \%$ of the inventoried species. Brachymenium columbicum (De Not.) Broth. represents a new record for the Cerrado phytogeographical domain.

The briocenological group with the greatest species richness was rupicolous (28 spp.), followed by corticicolous (14 spp.), epixilous (5 spp.), and terricolous (4 spp.) (Fig. 4). There were 16 species (42\%) with a preference for more than 1 type of substrate (polysubstrate) and 22 species $(58 \%)$ that colonized only 1 type of substrate (monosubstrate).

Diagnostic characterization of new records and remarkable species are provided here in alphabetical order. 
Table 1. List of species of acrocarpic moss found in CMNP. Dist. Pattern = Distribution pattern in Brazil, MO $=$ Moderately distributed, RE $=$ Restricted, $\mathrm{BR}=$ Broadly distributed; Phytog. Domain $=$ Phytogeographical domains, Amazon Forest $=\mathrm{AM}, \mathrm{Atlantic}$ Forest $=\mathrm{AT}$, Cerrado $=$ $\mathrm{CE}$, Caatinga $=\mathrm{CA}$, Pampa $=\mathrm{PA}$, Pantanal $=\mathrm{PL} ;{ }^{*}$ New record for the state of Maranhão; ${ }^{*}$ New record for the Northeast Region $(\mathrm{FBC}=\mathrm{Filipe}$ Bezerra Costa; $\mathrm{HCO}=$ Hermeson Cassiano de Oliveira; RRO = Regigláucia Rodrigues Oliveira).

\begin{tabular}{|c|c|c|c|c|c|}
\hline Taxon & $\begin{array}{c}\text { Dist. } \\
\text { pattern }\end{array}$ & $\begin{array}{l}\text { Phytog. } \\
\text { domain }\end{array}$ & Voucher & $\begin{array}{l}\text { Latitidue } \\
\text { (S) }\end{array}$ & $\begin{array}{l}\text { Longitude } \\
\text { (W) }\end{array}$ \\
\hline \multicolumn{6}{|l|}{ Bryophyta } \\
\hline \multicolumn{6}{|l|}{ Bartramiaceae } \\
\hline $\begin{array}{l}\text { Philonotis uncinata (Schwägr.) Brid. (Schwägrichen 1816, } \\
\text { Bridel 1827) }\end{array}$ & MO & $\mathrm{AM}, \mathrm{CA}, \mathrm{CE}, \mathrm{AT}$ & HCO 2708 (HABIT147) & $06^{\circ} 59^{\prime} 17^{\prime \prime} \mathrm{S}$ & $047^{\circ} 09^{\prime} 52^{\prime \prime}$ \\
\hline $\begin{array}{l}\text { * Philonotis sphaerocarpa (Hedw.) Brid. (Hedwig 1801, Bridel } \\
\text { 1827) }\end{array}$ & $\mathrm{BR}$ & $\begin{array}{l}\text { AM, CA, CE, AT, } \\
\quad \text { PA, PL }\end{array}$ & RRO 107 (HABIT153) & $06^{\circ} 59^{\prime} 17^{\prime \prime}$ & $047^{\circ} 09^{\prime} 52^{\prime \prime}$ \\
\hline \multicolumn{6}{|l|}{ Bryaceae } \\
\hline Bryum coronatum Schwägr. (Schwägrichen 1816) & BR & CE, AT & RRO148 (HABIT233) & $07^{\circ} 13^{\prime} 27^{\prime \prime}$ & $046^{\circ} 27^{\prime} 11^{\prime \prime}$ \\
\hline * Bryum limbatum Müll. Hal. (Müller 1851) & MO & AT & FBC $128(\mathrm{HABIT} 411)$ & $07^{\circ} 03^{\prime} 56^{\prime \prime}$ & $047^{\circ} 15^{\prime} 28^{\prime \prime}$ \\
\hline $\begin{array}{l}\text { * Brachymenium columbicum (De Not.) Broth. (Notaris 1859, } \\
\text { Brotherus 1903) }\end{array}$ & RE & $\begin{array}{l}\text { AM, } C A, C E, A T \\
\text { PA, PL }\end{array}$ & RRO 76 (HABIT85) & $07^{\circ} 01^{\prime} 28^{\prime \prime}$ & $047^{\circ} 02^{\prime} 52^{\prime \prime}$ \\
\hline \multicolumn{6}{|l|}{ Calymperaceae } \\
\hline * Calymperes afzelli Sw. (Swartz 1818) & MO & AT & RRO 380 (HABIT 757) & $07^{\circ} 04^{\prime} 25^{\prime \prime}$ & $047^{\circ} 19^{\prime} 30^{\prime \prime}$ \\
\hline * Calymperes erosum Müll. Hal. (Müller 1848) & $\mathrm{BR}$ & $A M, C E, A T$ & RRO 448 (HABIT975) & $07^{\circ} 07^{\prime} 53^{\prime \prime}$ & $047^{\circ} 25^{\prime} 48^{\prime \prime}$ \\
\hline Calymperes palisotii Schwägr. (Schwägrichen 1816) & $\mathrm{BR}$ & $\mathrm{AM}, \mathrm{CA}, \mathrm{CE}, \mathrm{AT}$ & FBC 208 (HABIT513) & $06^{\circ} 59^{\prime} 17^{\prime \prime}$ & $047^{\circ} 09^{\prime} 52^{\prime \prime}$ \\
\hline Octoblepharum albidum Hedw. (Hedwig 1801) & BR & $\mathrm{AM}, \mathrm{CE}, \mathrm{AT}$ & RRO 3 (HABIT 117) & $07^{\circ} 04^{\prime} 25^{\prime \prime}$ & $047^{\circ} 19^{\prime} 30^{\prime \prime}$ \\
\hline $\begin{array}{l}\text { * Octoblepharum cylindricum Schimp. ex Mont. (Montagne } \\
\text { 1840) }\end{array}$ & BR & $\mathrm{AM}, \mathrm{CA}, \mathrm{CE}, \mathrm{AT}$ & RRO 146 (HABIT189) & $07^{\circ} 13^{\prime} 28^{\prime \prime}$ & $046^{\circ} 27^{\prime} 11^{\prime \prime}$ \\
\hline $\begin{array}{l}\text { Octoblepharum pulvinatum (Dozy \& Molk.) Mitt. (Dozy \& } \\
\text { Molkenboer 1854, Mitten 1869) }\end{array}$ & $\mathrm{BR}$ & $\mathrm{AM}, \mathrm{CA}, \mathrm{CE}, \mathrm{AT}$ & RRO 381 (HABIT964) & $07^{\circ} 08^{\prime} 07^{\prime \prime}$ & $047^{\circ} 04^{\prime} 58^{\prime \prime}$ \\
\hline * Syrrhopodon ligulatus Mont. (Montagne 1856) & $\mathrm{BR}$ & $A M, C A, C E, A T, P L$ & RRO433 (HABIT972) & $07^{\circ} 07^{\prime} 53^{\prime \prime}$ & $047^{\circ} 25^{\prime} 48^{\prime \prime}$ \\
\hline * Syrrhopodon prolifer Schwägr. (Schwägrichen 1827) & $\mathrm{BR}$ & $\mathrm{AM}, \mathrm{CA}, \mathrm{CE}, \mathrm{AT}, \mathrm{PL}$ & RRO 379 (HABIT739) & $07^{\circ} 04^{\prime} 25^{\prime \prime}$ & $047^{\circ} 19^{\prime} 30^{\prime \prime}$ \\
\hline ** Syrrhopodon tortilis Hampe, (Hampe 1872) & BR & $\mathrm{AM}, \mathrm{CA}, \mathrm{CE}, \mathrm{AT}$ & RRO 27 (HABIT105) & $07^{\circ} 01^{\prime} 29^{\prime \prime}$ & $047^{\circ} 02^{\prime} 45^{\prime \prime}$ \\
\hline \multicolumn{6}{|l|}{ Dicranaceae } \\
\hline * Campylopus carolinae Grout (Grout 1939) & BR & $\mathrm{AM}, \mathrm{CE}, \mathrm{AT}, \mathrm{PL}$ & RRO 29 (HABIT273) & $07^{\circ} 13^{\prime} 27^{\prime \prime}$ & $046^{\circ} 27^{\prime} 11^{\prime \prime}$ \\
\hline Campylopus surinamensis Müll. Hal. (Müller 1848) & MO & $\mathrm{AM}, \mathrm{CE}, \mathrm{AT}$ & RRO 344 (HABIT680) & $07^{\circ} 04^{\prime} 25^{\prime \prime}$ & $047^{\circ} 19^{\prime} 30^{\prime \prime}$ \\
\hline $\begin{array}{l}\text { * Leucoloma tortellum (Mitt.) A.Jaeger (Mitten 1869, Jaeger } \\
\text { 1872) }\end{array}$ & MO & $\mathrm{AM}, \mathrm{CE}, \mathrm{AT}, \mathrm{PL}$ & FBC 25 (HABIT266) & $07^{\circ} 13^{\prime} 27^{\prime \prime}$ & $046^{\circ} 27^{\prime} 11^{\prime \prime}$ \\
\hline \multicolumn{6}{|l|}{ Fissidentaceae } \\
\hline Fissidens anguste-limbatus Mitt. (Mitten 1869) & MO & $\begin{array}{l}\text { AM, } C A, C E, A T \\
\quad P A, P L\end{array}$ & FBC 71 (HABIT218) & $07^{\circ} 13^{\prime} 27^{\prime \prime}$ & $046^{\circ} 27^{\prime} 11^{\prime \prime}$ \\
\hline Fissidens elegans Brid. (Bridel 1806) & BR & $\mathrm{AM}, \mathrm{CA}, \mathrm{CE}, \mathrm{AT}, \mathrm{PA}$ & RRO 39 (HABIT614) & $07^{\circ} 01^{\prime} 29^{\prime \prime}$ & $047^{\circ} 02^{\prime} 45^{\prime \prime}$ \\
\hline Fissidens flaccidus Mitt. (Mitten 1860) & $\mathrm{BR}$ & $\begin{array}{l}\mathrm{AM}, \mathrm{CA}, \mathrm{CE}, \mathrm{AT} \\
\mathrm{PA}, \mathrm{PL}\end{array}$ & RRO 280 (HABIT593) & $06^{\circ} 59^{\prime} 17^{\prime \prime}$ & $047^{\circ} 09^{\prime} 52^{\prime \prime}$ \\
\hline * Fissidens goyazensis Broth. (Brotherus 1895) & $\mathrm{BR}$ & $\begin{array}{l}\text { AM, } C A, C E, A T \\
\quad P A, P L\end{array}$ & RRO 39 (HABIT42) & $07^{\circ} 01^{\prime} 29^{\prime \prime}$ & $047^{\circ} 02^{\prime} 45^{\prime \prime}$ \\
\hline Fissidens guianensis Mont. (Montagne 1840) & BR & $\mathrm{AM}, \mathrm{CA}, \mathrm{CE}, \mathrm{AT}$ & RRO 70 (HABIT93) & $07^{\circ} 01^{\prime} 29^{\prime \prime}$ & $047^{\circ} 02^{\prime} 45^{\prime \prime}$ \\
\hline Fissidens hornschuchii Mont. (Montagne 1840) & RE & $\mathrm{CE}, \mathrm{AT}$ & RRO 447 (HABIT982) & $07^{\circ} 07^{\prime} 53^{\prime \prime}$ & $047^{\circ} 25^{\prime} 48^{\prime \prime}$ \\
\hline Fissidens neglectus H.A. Crum (Crum 1960) & BR & $\mathrm{CE}, \mathrm{AT}$ & RRO 142 (HABIT178) & $07^{\circ} 13^{\prime} 27^{\prime \prime}$ & $046^{\circ} 27^{\prime} 11^{\prime \prime}$ \\
\hline * Fissidens ornatus Herzog, (Herzog 1924) & BR & $\begin{array}{l}\text { AM, CA, CE, AT, } \\
\text { PA, PL }\end{array}$ & RRO 164 (HABIT206) & $07^{\circ} 13^{\prime} 27^{\prime \prime}$ & $046^{\circ} 27^{\prime} 11^{\prime \prime}$ \\
\hline * Fissidens pellucidus Hornsch., (Hornschuch 1840) & RE & $\begin{array}{l}\text { AM, CA, CE, AT, } \\
\text { PA, PL }\end{array}$ & RRO 165 (HABIT207) & $07^{\circ} 13^{\prime} 27^{\prime \prime}$ & $046^{\circ} 27^{\prime} 11^{\prime \prime}$ \\
\hline * Fissidens perfalcatus Broth. (Brotherus 1900) & MO & CE, AT & FBC 71 (HABIT284) & $07^{\circ} 13^{\prime} 27^{\prime \prime}$ & $046^{\circ} 27^{\prime} 11^{\prime \prime}$ \\
\hline ** Fissidens steeri Grout (Grout 1943) & $\mathrm{BR}$ & $\begin{array}{l}\text { AM, CA, CE, AT, } \\
\text { PA, PL }\end{array}$ & RRO 156 (HABIT 198) & $07^{\circ} 13^{\prime} 27^{\prime \prime}$ & $046^{\circ} 27^{\prime} 11^{\prime \prime}$ \\
\hline \multicolumn{6}{|l|}{ Leucobryacae } \\
\hline ** Leucobryum laevifolium Broth. (Brotherus 1901) & MO & AM, AT & FBC 48 (HABIT176) & $07^{\circ} 13^{\prime} 27^{\prime \prime}$ & $046^{\circ} 27^{\prime} 11^{\prime \prime}$ \\
\hline * Ochrobryum subulatum Hampe (Hampe 1897) & $\mathrm{BR}$ & $\mathrm{AM}, \mathrm{CE}$ & RRO 491 (HABIT974) & $07^{\circ} 07^{\prime} 53^{\prime \prime}$ & $047^{\circ} 25^{\prime} 48^{\prime \prime}$ \\
\hline Ochrobryum gardneri (Müll.Hal.) Mitt. (Müller 1844, Mitten 1869) & $\mathrm{BR}$ & $A M, C E, A T, P L$ & RRO 211 (HABIT347) & $06^{\circ} 58^{\prime} 59^{\prime \prime}$ & $047^{\circ} 22^{\prime} 43^{\prime \prime}$ \\
\hline \multicolumn{6}{|l|}{ Pottiaceae } \\
\hline * Barbula indica (Hook.) Spreng. (Hooker 1819, Sprengel, 1824) & BR & $\mathrm{AM}, \mathrm{CA}, \mathrm{CE}, \mathrm{AT}$ & RRO 196 (HABIT361) & $06^{\circ} 58^{\prime} 59^{\prime \prime}$ & $047^{\circ} 22^{\prime} 43^{\prime \prime}$ \\
\hline Hyophilla involuta (Hook.) A. Jaeger (Hooker 1819, Jaeger 1873) & BR & $\begin{array}{l}\text { AM, } C A, C E, A T \\
\text { PA, PL }\end{array}$ & RRO 231 (HABIT403) & $06^{\circ} 58^{\prime} 59^{\prime \prime}$ & $047^{\circ} 22^{\prime} 43^{\prime \prime}$ \\
\hline $\begin{array}{l}\text { Hyophiladelphus agrarius (Hedw.) RH Zander (Hedwig 1801, } \\
\text { Zander 1995) }\end{array}$ & BR & $\begin{array}{l}\text { AM, CA, CE, AT, } \\
\quad \text { PA, PL }\end{array}$ & RRO 140 (HABIT174) & $07^{\circ} 13^{\prime} 27^{\prime \prime}$ & $046^{\circ} 27^{\prime} 11^{\prime \prime}$ \\
\hline $\begin{array}{l}\text { Plaubelia sprengelii (Schwägr.) RH Zander (Schwägrichen } \\
\text { 1823, Zander 1993) }\end{array}$ & BR & $\mathrm{AM}, \mathrm{CA}, \mathrm{CE}, \mathrm{AT}$ & RRO 346 (HABIT683) & $07^{\circ} 04^{\prime} 25^{\prime \prime}$ & $047^{\circ} 19^{\prime} 29^{\prime \prime}$ \\
\hline * Trichostomum brachydontium Bruch, (Bruch 1829) & MO & AT & FBC 381 (HABIT773) & $07^{\circ} 04^{\prime} 42^{\prime \prime}$ & $047^{\circ} 05^{\prime} 43^{\prime \prime}$ \\
\hline $\begin{array}{l}\text { * Trichostomum tenuirostre (Hook. \& Taylor) Lindb. (Hooker \& } \\
\text { Taylor 1827, Lindberg 1864) }\end{array}$ & $\mathrm{BR}$ & $\mathrm{AM}, \mathrm{CA}, \mathrm{CE}, \mathrm{AT}$ & RRO 462 (HABIT1048) & $07^{\circ} 07^{\prime} 53^{\prime \prime}$ & $047^{\circ} 25^{\prime} 48^{\prime \prime}$ \\
\hline $\begin{array}{l}\text { ** Tortella tortuosa (Schrad. ex Hedw.) Limpr. (Hedwig 1801, } \\
\text { Limpricht 1888) }\end{array}$ & RE & AT & RRO 104 (HABIT129) & $06^{\circ} 59^{\prime} 17^{\prime \prime}$ & $047^{\circ} 09^{\prime} 52^{\prime \prime}$ \\
\hline
\end{tabular}



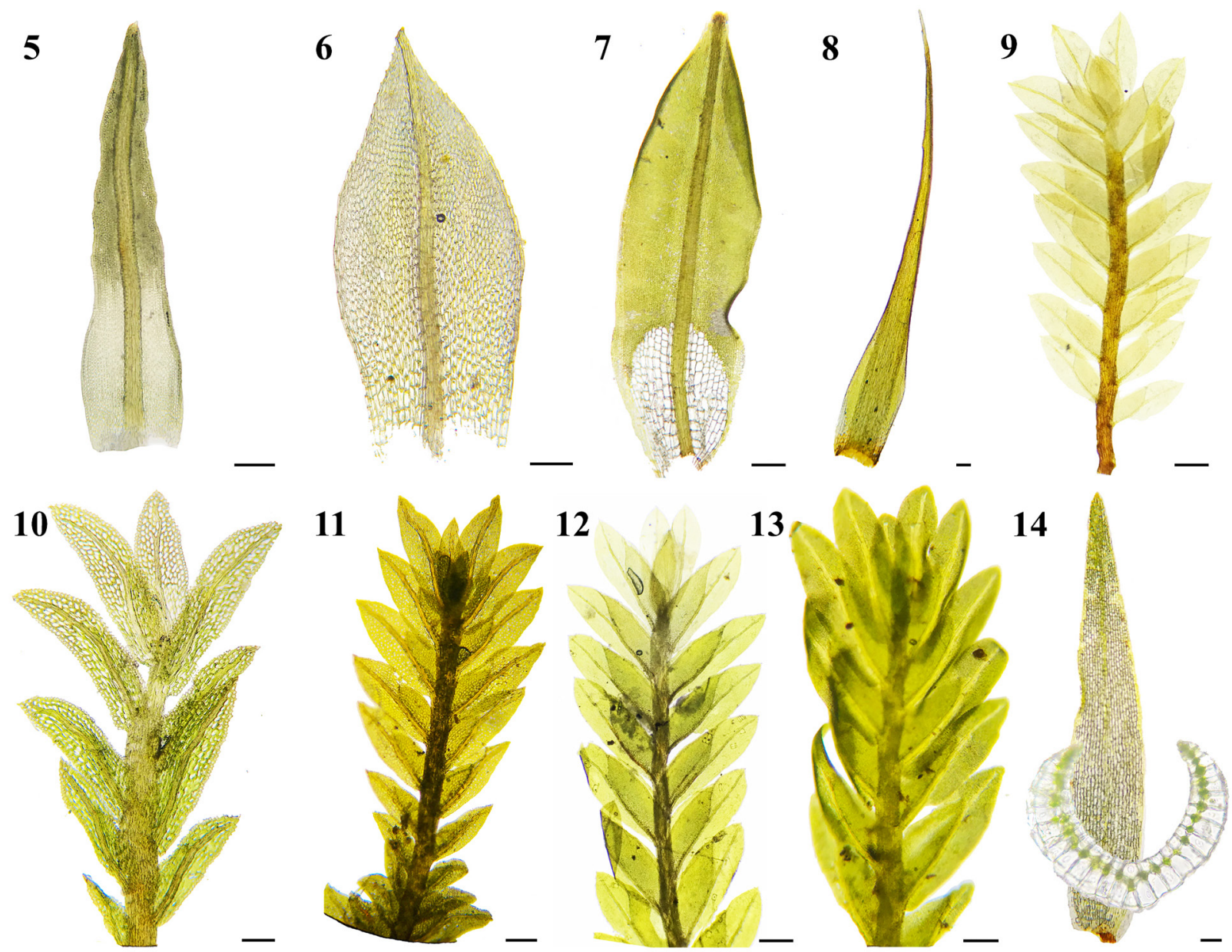

Figure 5-14. Acrocarpic mosses of Chapada das Mesas National Park. 5. Barbula indica (Hook.) Spreng. 6. Bryum limbatum Müll. Hal. 7. Calymperes afzelii Sw. 8. Campylopus carolinae Grout. 9. Fissidens goyazensis Broth. 10. Fissidens ornatus Herzog, 11. Fissidens pellucidus Hornsch. 12. Fissidens perfalcatus Broth. 13. Fissidens steeri Grout. 14. Leucobryum laevifolium Broth. Scale bar $=100 \mu \mathrm{m}$.

\section{Barbula indica (Hook.) Spreng. (Fig. 5)}

Recognized by oval to elliptical leaves; margins plane or weakly recurved at mid-leaf; apex acute to rounded, costae percurrent or subpercurrent, laminal cells quadrate and strongly pluripapillose. Habitat: soil and rocks. Brazilian range: AC, AM, PA, BA, PE, RN, SE, DF, GO, MS, MT, ES, MG, RJ, SP, PR, RS.

\section{Brachymenium columbicum (De Not.) Broth.}

Recognized by oblong-lanceolate leaves, acute apex, entire margins, short-excurrent costae, elongate-rhomboidal cells in upper half and mid region, while quadratic cells at base. Habitat: rocks. Brazilian range: CE, SP.

\section{Bryum limbatum Müll. Hal. (Fig. 6)}

Recognized by ovate to oblong leaves, apex broadly acute, entire to distally serrate margins, border with long-rectangular cells with thick walls, costae percurrent, basal cells of lamina rectangular and thin-walled. Habitat: soil. Brazilian range: DF, MS, ES, MG, RJ, SP, PR, RS, SC.

\section{Calymperes afzelli Sw. (Fig. 7)}

Recognized by cancellinae ending in acute angles, costae percurrent to long-excurrent, spiny-papillose costa on adaxial surface, many propagules at the tip of costa.
Habitat: rocks. Brazilian range: $\mathrm{AC}, \mathrm{AM}, \mathrm{AP}, \mathrm{RO}, \mathrm{RR}$, TO, BA, PB, PE, MS, MT, ES, RJ, SP, SC.

\section{Calymperes erosum Müll. Hal.}

Species close to C. afzelli but differs in having smooth costae on adaxial surface. Habitat: tree trunks. Brazilian range: $\mathrm{AC}, \mathrm{AM}, \mathrm{AP}, \mathrm{PA}, \mathrm{RO}, \mathrm{RR}, \mathrm{BA}, \mathrm{PB}, \mathrm{PE}, \mathrm{GO}, \mathrm{MT}$, MG, ES, RJ.

\section{Campylopus carolinae Grout (Fig. 8)}

Recognized by hyaline hair point, basal portion of leaves hyaline and strong transition of ribbed costae with lamina in cross section. Habitat: soil and rocks. Brazilian range: $\mathrm{RO}, \mathrm{RR}, \mathrm{BA}, \mathrm{DF}, \mathrm{MT}, \mathrm{MS}, \mathrm{MG}, \mathrm{SP}, \mathrm{PR}$.

\section{Fissidens goyazensis Broth. (Fig. 9)}

Recognized by limbate and imbricate leaves, unipapillose cells and axillary hyaline nodules. Habitat: rocks. Brazilian range: $\mathrm{AM}, \mathrm{BA}, \mathrm{CE}, \mathrm{PB}, \mathrm{PE}, \mathrm{PI}, \mathrm{DF}, \mathrm{GO}, \mathrm{MG}$, RJ, SP.

\section{Fissidens ornatus Herzog (Fig. 10)}

Recognized by margin with hexagonal cells, similar in shape to median cells but smaller. Habitat: rocks. Brazilian range: $\mathrm{AC}, \mathrm{AM}, \mathrm{PA}, \mathrm{RO}, \mathrm{RR}, \mathrm{BA}, \mathrm{PE}, \mathrm{DF}, \mathrm{MT}$, MG, SP. 


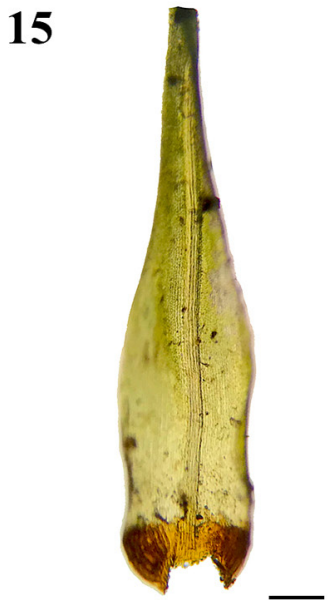

20

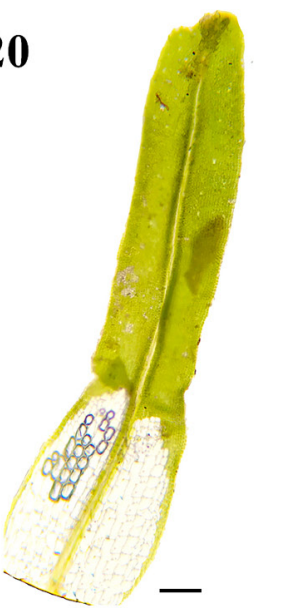

16

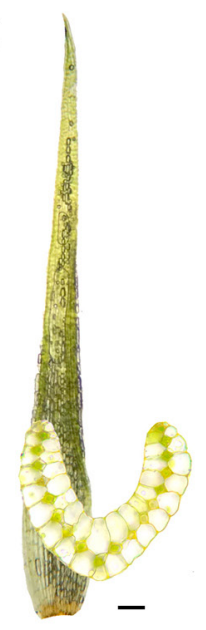

17

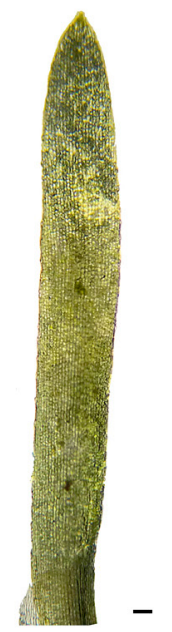

\section{1}

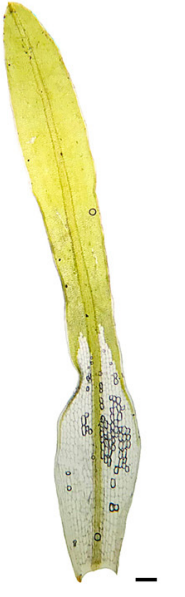

22
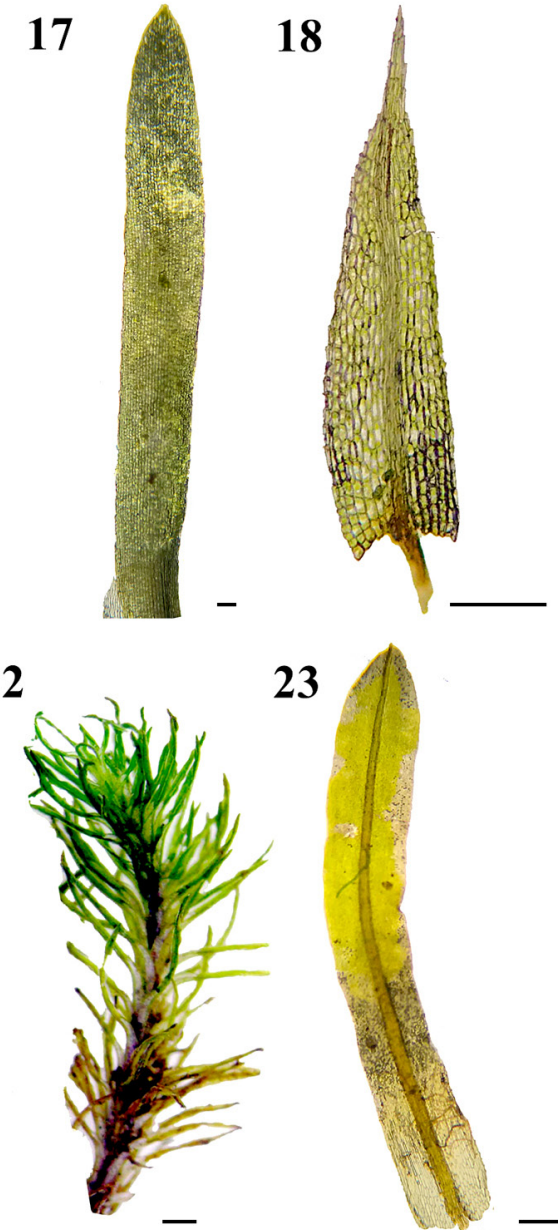

18

19

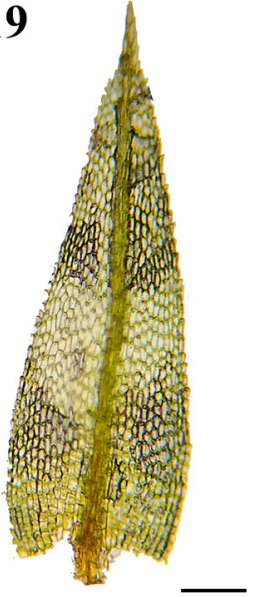

23

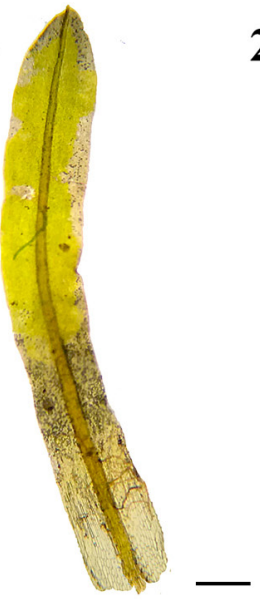

24

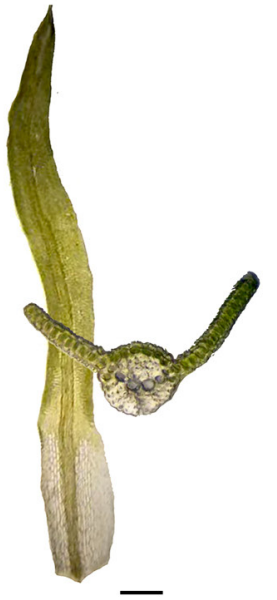

Figure 15-24. Acrocarpic mosses of Chapada das Mesas National Park 15. Leucoloma tortellum (Mitt.) A. Jaeger. 16. Ochrobryum subulatum Hampe. 17. Octoblepharum cylyndricum Mont. 18. Philonotis sphaerocarpa (Hedw.) Brid., 19. Philonotis uncinata (Schwägr.) Brid. 20. Syrrhopodon ligulatus Mont. 21. Syrrhopodon prolifer Schwägr. 22. Syrrhopodon tortilis Hampe. 23. Trichostomum tenuirostre (Hook. \& Taylor) Lindb. 24. Tortella tortuosa (Hedw.) Limpr. Scale bar $=100 \mu \mathrm{m}$.

\section{Fissidens pellucidus Hornsch. (Fig. 11)}

Recognized by gutulate and irregular quadratic hexagonal laminal cells. Habitat: rocks. Brazilian range: AC, AM, PA, RO, RR, TO, BA, CE, PB, PE, DF, GO, MG, ES, MG, RJ, SP, PR, RS, SC.

\section{Fissidens perfalcatus Broth. (Fig. 12)}

Recognized by strongly unipapillose laminal cells and limbidium restricted to vaginant lamina. Habitat: rocks. Brazilian range: TO, BA, PB, PE, GO, MT, ES.

Fissidens steeri Grout. (Fig. 13)

Recognized by strongly pluripapillose cells with bifurcated costae at end. Habitat: rocks. Brazilian range: GO, SP.

\section{Leucobryum laevifolium Broth. (Fig. 14)}

Plants whitish to pale green in color, leaves grouped, costa reaching almost the leaf width, percurrent, smooth; outer walls of cells convex in crosssection of the apex region. Straight leaves differentiate this species from $L$. martianum, which has falcate leaves. Habitat: roots and tree trunks. Brazilian range: AM, MG, MT, RJ, RS, SC, SP.

\section{Leucoloma tortellum (Mitt.) A. Jaeger (Fig. 15)}

Distinguished by the long-lanceolate leaf, with oval base, entire margin, crenulated in apical region, apex acute to obtuse, costae strong, upper cells pluripapillose, elongated at margin. Alar cells long-rectangular, smooth, inflated, forming auricles. Habitat: rocks. Brazilian range: AM, CE, MG, PA, RO, RR.

\section{Ochrobryum subulatum Hampe (Fig. 16)}

Plants small, forming lax to dense tufts, yellowish green in color. Differs from O. gardneri, the most common species of the genus, because of its linear lanceolate leaves with long acuminate apexes. Habit: rocks and tree trunks. Brazilian range: AC, AM, PA, RO, RR, TO, AL, PE, DF, GO, MT, SP.

\section{Octoblepharum cylyndricum Mont. (Fig. 17)}

Differs from $O$. albidum Hedw., the most common species of the genus, by its longer setae, oblong-cylindrical capsules, and acute to gradually apiculate leaves that are entire at the apex. Habitat: tree trunks. Brazilian range: $\mathrm{AC}, \mathrm{AM}, \mathrm{AP}, \mathrm{PA}, \mathrm{RO}, \mathrm{RR}, \mathrm{TO}, \mathrm{BA}, \mathrm{CE}, \mathrm{PB}, \mathrm{PI}$, RN, SE, DF, GO, MS, MG, ES, MG, SP.

\section{Philonotis sphaerocarpa (Hedw.) Brid. (Fig. 18)}

Recognized by salient costae on adaxial surface, acuminate apex and small cells at base of lamina. Growing 
in tufts on rocky cliffs and banks of waterfalls. This is the first citation for the state of Maranhão. Habitat: rocks. Brazilian range: AM, BA, CE, MT, RJ, SC, SP.

\section{Philonotis uncinata (Schwägr.) Brid. (Fig. 19)}

Lanceolate leaves, toothed margin in apical region, apex acuminate, coast strong, subpercurrent, cells of the superior portion of the leaf quadratic-rectangular, with papillae at the apexes, at base smaller, irregularly quadratic, smooth. Habitat: rocks. Brazilian range: $\mathrm{AC}, \mathrm{AM}, \mathrm{CE}, \mathrm{BA}, \mathrm{DF}, \mathrm{MG}, \mathrm{MT}$, MS, PA, PR, PE, PI, RJ, RS, RO, SP.

\section{Syrrhopodon ligulatus Mont. (Fig. 20)}

Recognized by leaves with ligulate apexes, pluripapillose cells above shoulders, margins bordered at and just above shoulders. Habitat: roots. Brazilian range: AC, AM, AP, PA, RO, RR, BA, PE, DF, GO, MS, MG, RJ, SP.

\section{Syrrhopodon prolifer Schwägr. (Fig. 21)}

Plants dark-green in color, straight contorted when dry, linear-lingulate blades, margins entire at base, costa often spinose near leaf apex. Habitat: soil and rocks. Brazilian range: $\mathrm{AC}, \mathrm{AM}, \mathrm{AP}, \mathrm{PA}, \mathrm{RO}, \mathrm{TO}, \mathrm{AL}, \mathrm{BA}, \mathrm{CE}, \mathrm{PE}$, PI, SE, DF, GO, MT, ES, MG, RJ, SP, PR, RS, SC.

\section{Syrrhopodon tortilis Hampe (Fig. 22)}

Similar to $S$. prolifer and often misidentified as such. Distinguishable by red to dark green color, wide lamina and cancelinae cells forming a concave region that always produces a wide-spreading habit to the leaves. Habitat: decomposing tree trunks and rocks. Brazilian range: $\mathrm{MG}$, RJ, SP, RS, SC.

\section{Trichostomum brachydontium Bruch.}

Highly variable with leaves sometimes being slender with a tapering tip. Leaves held at an angle of up to $45^{\circ}$ or are recurved away from stem when moist, but crisp and incurved when dry, margins normally plane, but may be narrowly recurved. Habitat: decomposing tree trunks and rocks. Brazilian range: $\mathrm{PA}, \mathrm{RO}, \mathrm{BA}, \mathrm{PE}, \mathrm{ES}, \mathrm{PR}$.

Trichostomum tenuirostre (Hook. \& Taylor) Lindb. (Fig. 23)

Shoot tips usually dull green in color, and brown or almost black below. Leaves spread and wavy, crisp when dry, tips usually acute and margins plane. The species is very similar in appearance to $T$. brachydontium, but T. brachydontium is typically yellow-green in color and often grows in drier places. Habitat: rocks and tree trunks. Brazilian range: AM, PA, RO, RR, BA, CE, PI, SE, DF, GO, MT, MG, SP, RS.

\section{Tortella tortuosa (Hedw.) Limpr. (Fig. 24)}

Leaves long, linear, crisp when dry, margins strongly undulate, cells at base differentiated, long, hexagonal and hyaline, subquadratic above, pluripapillose, costa percurrent. Habitat: rocks. Brazilian range: RJ, RS.

\section{Discussion}

The species richness of acrocarpic mosses found in the CMNP is highly consistent with published information on the bryophyte flora of the Cerrado. There is a high diversity of species in this phytogeographical domain, as found by Câmara (2008) who recorded 26 species of acrocarpic mosses in a survey in the IBGE Ecological Reserve in Brasília, Distrito Federal. Carmo and Peralta (2016) found 28 species of mosses, including 16 acrocarpic species, in a study in Serra da Canastra National Park. In another study, Rios et al. (2016) found 16 species in an area between several Cerrado physiognomies in the state of Goiás. In the Cerrado of Maranhão state, Oliveira et al. (2018) found 20 species of acrocarpic mosses. Our results, as well as those of previous studies, demonstrate the high species richness of acrocarpic mosses in the Cerrado and the high floristic potential of bryophyte species in CMNP. The importance of floristic surveys in conservation is reinforced. Floristic analysis allows comparisons within and between forest formations in space and time, generate data on the richness and diversity of an area, as well as enable the formulation of theories, test hypotheses, and produce results that will serve as the basis for other studies (Myers et al. 2000). In this way, with our results, we hope to collaborate with the management of the CMNP.

Calymperaceae was one of the most representative families, which can be explained by the broad distribution of this family in the tropics (Gradstein et al. 2001). The high representativeness of this family is demonstrated in floristic surveys at tropical region (Cerqueira et al. 2015, Oliveira et al. 2018). The composition being dominated by Acrocarpic mosses dominate the bryophyte flora on rocky outcrops (Silva and Germano 2013, Silva et al. $2014 \mathrm{a}, 2014 \mathrm{~b}$ ), where roughly $74 \%$ of the species are acrocarpic. The most species-rich families in the studies by Silva and Germano (2013) and Silva et al. (2014a, 2014b) were Leucobryaceae, Bryaceae, and Pottiaceae; in our study, these families were also highly species rich, which reflects the great availability in the study area of exposed habitats, where these families are typically found. These families are adapted for sunny substrates (Richards 1984).

The large number of species in our study belonging to the rupicolous bryocenological group is correlated to the availability of rocky substrata near waterfalls, where there are numerous rocky cliffs. Our results corroborate the findings of Carmo and Peralta (2016). The predominance of rupicolous community contrasts with tropical forests where corticicolous bryophytes predominate (Richards 1984, 1988, Gradstein and Pócs 1989).

In CMNP, $57 \%$ of the bryophyte species are acrocarpic species. Most of the acrocarpic moss species found, 25 species or, are broadly distributed (Table 1). These species (e.g., Hyophila involuta, Octoblepharum albidum, and Calymperes palisotii) are generalists and can easily occupy several types of substrates.

Nine species are moderately distributed (24\%) and 4 species are restricted (10\%). The occurrence of these 4 species reinforces the importance of CMNP as a conservation unit with diverse vegetation types in Cerrado 
and areas transitioning to Amazon Forest and Caatinga. The rich mosses flora is favored by conditions, such as humidity and a variety of substrates.

\section{Aknowledgements}

We are grateful to the Instituto Chico Mendes de Conservação da Biodiversidade (ICMBio) for the collecting permit and for logistical support. RRO thanks Fundação de Amparo à Pesquisa e Desenvolvimento Científico e Tecnológico do Maranhão (FAPEMA) and Coordenação de Aperfeiçoamento de Pessoal de Nível Superior (CAPES) for the scholarship tendered. Thank you to the reviewers who provided helpful comments and made valuable suggestions to improve the manuscript.

\section{Authors' contributions}

RRO collected the data, made identifications and wrote the text; HCO and GMC collected the data and reviewed the text; DFP confirmed the identifications and reviewed the text.

\section{References}

Bordin J, Yano O (2013) Fissidentaceae (Bryophyta) do Brasil. Boletim do Instituto de Botânica 22: 1-168.

Brotherus VF (1895) Nouvelles contributions à la flore bryologique du Brésil. Bihang til kongliga Svenska vetenskaps-akademiens handlingar 21: 3-76.

Brotherus VF (1900) Die Laubmoose der ersten Regnellichen Expedition. Bihang till kongliga Svenska vetenskaps akademiens handlingar 26: 1-65

Brotherus VF (1901) Musci in report on two botanical collections made by Messrs. F.V. McConnell and J.J. (ed.) Quelch at Mount Roraima in British Guiana. Transactions of the Linnean Society of London, Botany (2nd Series): 6: 1-90.

Brotherus VF (1903) Musci (Laubmoose). III Unterklasse Bryales: II Spezieller Teil, Gruppe I. Acrocarpi. In: Engler A, Prantl K (Eds) Die natürlichen Pflanzenfamilien: insbesondere den Nutzpflanzen, unter Mitwirkung zahlreicher hervorragender Fachgelehrten begründet. Teil 3. Engelmann, Leipzig, 481-528. https://doi. org/10.5962/bhl.title.4635

Brotherus VF (1910). Neue Laubmoosgattungen. Öfversigt af Finska Vetenskaps-Societetens Förhandlingar 52A: 1-1910.

Bridel S (1806-1812) Muscologiae recentiorum supplementum, vol. 1. C.G. Ettinger, Gotha, 167 pp. https://doi.org/10.5962/bhl.title.20

Bridel S (1827) Bryologia universa seu systematica ad novam methodum dispositio: historia et descriptio omnium muscorum frondosorum huscusque cognitorum cum synonymia ex auctoribus probatissimis. Pars II. J.A. Barth, Leipzig, 856 pp. https://doi. org/10.5962/bhl.title.60260

Bruch P (1829) Flora oder Botanische Zeitung: welche Recensionen, Abhandlungen, Aufsätze, Neuigkeiten und Nachrichten, die Botanik betreffend, enthält/herausgegeben von der Königl. Botanischen Gesellschaft in Regensburg 12: 1-393.

Buck WR (1998) Pleurocarpous Mosses of the West Indies. Memoirs of the New York Botanical Garden 1: 1-401.

Carmo DM, Peralta DF (2016) Survey of bryophytes in Serra da Canastra National Park, Minas Gerais, Brazil. Acta Botanica Brasilica 30 (2): 254-265. https://doi.org/10.1590/010233062014abb3608

Câmara PEAS (2008) Musgos acrocárpicos das Matas de Galeria da Reserva Ecológica do IBGE, RECOR, Distrito Federal, Brasil. Acta Botânica Brasilica 22: 1027-1035. https://doi.org/10.1590/
S0102-33062008000400013

Cerqueira GR, Ilkiu Borges AL, Manzatto, AG, Maciel, S. (2015) Briófitas de um fragmento de floresta ombrófila aberta no município de Porto Velho e novas ocorrências para Rondônia, Brasil. Biota Amazônia 5 (2): 1-75. https://doi.org/10.18561/21795746/biotaamazonia.v5n2p $71-5$

Costa DP, Pôrto KC, Luizi-Ponzo AP, Ilkiu-Borges AL, Bastos CJP, Câmara PEAS, Peralta DF, Bôas-Bastos SBV, Imbassahy CAA, Henriques DK, Gomes HCS, Rocha LM, Santos ND, Siviero TS, Vaz-Imbassahy TF, Churchill SP (2011) Synopsis of the Brazilian moss flora: checklist, distribution and conservation. Nova Hedwigia 93: 277-334. https://doi.org/10.1127/0029-5035/2011/0093-0277

Costa DP, Peralta DF (2015) Bryophytes diversity in Brazil. Rodriguésia 66(4): 1063-1071. https://doi.org/10.1590/2175-7860201566409

Crum HA (1960) Three news species of Fissidens. The Bryologist 63: 93-98. https://doi.org/10.2307/3240879

Dozy F, Molkenboer HJ (1854) Musci. In: Zollinger H (Ed.) Systematisches Verzeichnis der im indischen Archipel in den Jahren gesammelten sowie der aus Japan empfangenen Pflanzen 1, E. Kiesling, Zürich, 22-33.

Forzza RC, Leitman PM, Costa AF (2010) Introdução. In: Forzza RC, Baumgratz JFA, Bicudo CEM, Carvalho Jr. AA, Costa A, Costa DP, Hopkins M, Leitman PM, Lohmann LG, Maia LC, Martinelli G, Menezes M, Morim MP, Coelho MAN, Peixoto AL, Pirani JR, Prado J, Queiroz LP, Souza VC, Stehmann JR, Sylvestre LS, Walter BMT, Zappi D (Eds) Catálogo de Plantas e Fungos do Brasil. Vol. 1. Rio de Janeiro, Jardim Botânico do Rio de Janeiro, 875 pp .

Frahm JP (2003) Manual of Tropical Bryology. Tropical Bryology 23: 1-196. https://doi.org/10.11646/bde.23.1.1

Frahm JP (1991) Dicranaceae: Campylopodioideae, Paraleucobryoideae. Flora Neotropica Monograph 54: 1-237.

Galinkin M, Dias A, Latrubesse EM, Scardua FP, Mendonça AF, Arruda MB (2004) Projeto Corredor Ecológico Araguaia-Bananal. In: Arruda,MB; Sá LFSN (Eds) Corredores Ecológicos-Uma abordagem integradora de ecossistemas no Brasil. Ed. IBAMA, Brasília, 1: $81-132$.

Gradstein SR, Pócs T (1989) Bryophytes. In: Lieth H, Werger MJA. (Eds) Tropical Rain Forest Ecosystems. Elsevier, Amsterdan, 311-325.

Gradstein SR, Churchill SP, Salazar-Allen N (2001) Guide to the bryophytes of tropical America. Memoirs of the New York Botanical Garden 1: 1-577.

Goffinet B, Buck WR, Shaw AJ (2009) Morphology, anatomy and classification of the Bryophyta. In: Goffinet B, Shaw AJ (Eds) Bryophyte Biology, 2nd edition. Cambridge University Press, Cambridge, 56-138. https://doi.org/10.1017/CBO9780511754807.003

Grout AJ (1936-1939) Moss Flora of North America. Vol. 1. Privately published by the author, Newfane, Vermont, 1-264.

Grout AJ (1943) Fissidentaceae. In Britton NL (Ed.) North American Flora. Vol. 15. New York Botanical Garden, Bronx, 167-202.

Hampe E (1897) Revision du genre Ochrobryum. Journal de Botanique (Morot) 11: 138-153.

Hampe E (1872) Musci frondosi. Symbolae ad floram Brasiliae centrales cognoscendam. Videnskabelige meddelelser fra dansk naturhistoriske Forening i Kjöbenhavn (serie 3) 10: 36-59.

Hampe E (1897) Revision du genre Ochrobryum. Journal de Botanique (Morot) 11: 138-153.

Hedwig J (1801) Species muscorum frondosorum: descriptae et tabulis aeneis lxxvii coloratis illustratae. Barthius, 1-353. https://doi. org/10.5962/bhl.title.26

Herzog T (1924) Contribuições ao conhecimento da flora bryologica do Brasil. Archivos de Botanica do Estado de São Paulo 2: 27-105.

Hooker WJ (1818-1820) Musci Exotici: Containing Figures and Descriptions of New or Little Known Foreign Mosses and other Cryptogamic Subjects. Vol. II. in Longman, Hurst, Rees, Orme, and Brown, London, 1-135. https://doi.org/10.5962/bhl.title.10721

Hooker WJ, Taylor T (1827) Muscologia Britannica, Second Edition, Corrected and Enlarged Longman, Rees, Orme, Brown \& Green, London, 1-272. 
Hornschuch F (1840) Musci. In: Martius CFP, Eichler AW, Endlicher IL, Fenzl E, Mary B, Oldenburg R, Urban I (Eds.) Flora Brasiliensis enumeratio plantarum in Brasilia hactenus detectarum quas suis aliorumque botanicorum studiis descriptas et methodo naturali digestas partim icone illustratas. Flora Brasiliensis 1 (2): 1-172. https://doi.org/10.5962/bhl.title.454

Jaeger A (1871-1873) Adumbratio flore muscorum totius orbis terrarum. Part 7. Bericht über die Thätigkeit der St. Gallischen Naturwissenschaftlichen Gesellschaft während des Vereinsjahres 1: 1-202.

La Farge-England C (1996) Growth form, branching pattern, and perichaetial position in mosses: cladocarpy and pleurocarpy redefined. The Bryologist 99: 170-186. https://doi.org/10.2307/3244546

Lindberg GA (1864) Uppställning af familjen Funariaceae. Öfversigt af Förhandlingar: Kongl. Svenska Vetenskaps-Akademien 10: 589-608.

Luizi-ponzo AP, Siviero TS, Amorim ET, Henriques DK, Rocha LM, Gomes HC, Paiva LA, Rodrigues RS, Silva GD, Ribeiro GC, Gomes C, Campeão AS (2013) Briófitas do Parque Estadual do Ibitipoca (Minas Gerais, Brasil). In: Forzza RC, Neto LM, Salimena FRG, Zappi D (Eds) Flora do Parque Estadual do Ibitipoca e seu entorno. Universidade Federal de Juiz de Fora, Juiz de Fora, 95-122.

Marques AR, Amorim MCCT (2014) Saberes Geográficos Integrados Aos Estudos Territoriais sob a ótica da Implantação do Parque Nacional Da Chapada das Mesas, Sertão de Carolina/MA. Geografia em Questão 7 (2): 100-117.

Martins FP, Salgado AAR, Barreto HR (2017) Morfogênese da Chapada das Mesas (Maranhão Tocantins): paisagem cárstica e poligenética Revista Brasileira de Geomorfologia 3: 1-114.

Medeiros, JD (2011) Guia de campo: vegetação do Cerrado 500 espécies. Ministério do Meio Ambiente, Série Biodiversidade 43 $1-532$.

Lima LP, Tchaicka L, Avelar, JJC, Cunha AMC (2007) Plano operativo de Prevenção e Combate aos Incêndios Florestais do Parque Nacional da Chapada das Mesas. Ministério do Meio Ambiente, Instituto Chico Mendes de Conservação da Biodiversidade, Carolina, 1-18.

Mitten W (1860) Hepaticae Indiae Orientalis; an enumeration of the species of the Hepaticae of East Indies. Journal of the Proceedings Linnean Society of London, Botany 5: 89-108. https://doi. org/10.1111/j.1095-8312.1860.tb01045.x

Mitten W (1869) Musci austro-americani. Journal of the Proceedings of the Linnean Society, Botany 12: 1-659.

Montagne JFC (1840) Seconde Centurie de plants cellulaires exotiques nouvelles. Décades VI, VII et VIII. Journal of the Proceedings of the Linnean Society, Botany 14: 331-337.

Montagne JFC (1856) Huitième centurie de plantes cellulaires nouvelles tant indigènes qu'exotiques, decades I à III. Annales des sciences naturelles, botanique 6: 179-199.

Moraes RC, Lima LP (2007) Utilização de SIG como ferramenta na gestão do Parque Nacional Chapada das Mesas (Carolina/MA) Simpósio Brasileiro de Sensoriamento Remoto, Florianopólis, Brasil, 1: 4057-4064.

Müller C (1844) Beiträge zu einer Flora Cryptogamica Oldenburgensis. Botanische Zeitung, Berlin, 2: 1-741.

Müller C (1846) Nachträgliche Bemerkungen über die von Gardner in Brasilien gesammelten Laubmoose. Botanische Zeitung 3 $105-111$.

Müller C (1848) Plantae Kegelinae Surinamenses: musci frondosi. Linnaea 21: 181-200.

Müller C (1851) Synopsis Muscorum Frondosorum omnium hucusque Cognitorum. Alb. Förstner, Berlin, 1: 511-772. https://doi.org/ 10.5962/bhl.title.31

Myers N, Mittermeier RA, Mittermeier CG, Fonseca GAB, Kent J (2000) Biodiversity hotspots for conservation priorities. Nature 403: 853-858. https://doi.org/10.1038/35002501

Notaris JD (1859) Musci Napoani sive muscorum ad Flumen Napo in Columbia. Memorie della Reale Accademia delle Scienze di Torino 18: $437-455$
Oliveira RF, Silva GS, Oliveira RR, Oliveira HC, Coneição GM (2018) Musgos (Bryophyta) de um fragmento do cerrado maranhense, Brasil. Biota Amazônia 8: 12-18. https://doi.org/10.18561/21795746/biotaamazonia.v8n2p12-18

Peralta DF, Brito ES, Varão LF, Conceição, GM, Cunha, IPR (2011) Novas Ocorrências e Lista de Briófitas do Estado do Maranhão, Brasil. Pesquisa em Foco 19: 63-78.

Richards WP (1984) The ecology of tropical forest bryophytes. In: Schuster RM (Ed) New Manual of Bryology. Vol. 2. Hattori Botanical Laboratory, Nichinan, 1233-1270.

Richards WP (1988) Tropical forest bryophytes. Synusiae and strategies. The Journal Hattori Botanical Laboratory 64: 1-4.

Rios ABM, Oliveira JPS, Silva RP, Neto JFO, Oliveira LS, Peralta DF, Maccagnan DHB (2016) Bryophyte diversity in an area of Brazilian Cerrado in Central West. Neotropical Biology and Conservation 11 (3): 132-140.

Robbins RG (1952) Bryophyte ecology of a dune area in New Zealand. Vegetatio, Acta Geobotanica 4: 1-31.

Schwaegrichen CF (1816) Species Muscorum Frondosorum, Supplementum Primum. Barth, Leipzig, 60-61.

Schwaegrichen CF (1823) Species Muscorum Frondosorum, Supplementum Secundum. Barth, Leipzig, 1: 1-86.

Schwaegrichen CF (1827) Species Muscorum Frondosorum, Supplementum Secundum. Barth, Leipzig, 2: 1-99.

Sharp AJ, Crum H, Eckel P (1994) The moss flora of Mexico. Memoirs of the New York Botanical Garden 69: 1-1113.

Schimper WP (1840) Plantes cellulaires exotiques. Annales des Sciences Naturelles, Botanique 14: 1-349.

Silva JB, Germano SR (2013) Bryophytes on rocky outcrops in the caatinga biome: a conservationist perspective. Acta Botanica Brasilica 27: 827-835. https://doi.org/10.1590/S010233062013000400023

Silva JB, Santos ND, Pôrto KC (2014a) Beta diversity: effect of geographical distance and environmental gradients on the rocky outcrop bryophytes. Cryptogamie Bryologie 35: 133-163. https://doi. org/10.7872/cryb.v35.iss2.2014.133

Silva TO, Silva MPP, Pôrto KC (2014b) Briófitas de afloramentos rochosos do estado de Pernambuco, Brasil. Boletim do Museu de Biologia Mello Leitão 36: 85-100.

Sprengel C (1821-1824) Nomenclator botanicus: enumerans ordine alphabetico nomina atque synonyma, tum generica tum specifica, et a Linnaeo et recentioribus de re botanica scriptoribus plantis phanerogamis imposita, 2. Stuttgart \& Tubingen, Steudel, 1-72.

Swartz O (1818-1820) Calymperes, eine neue Moosgattung in Jahrbücher der Gewächskunde 1: 1-5

Valente EB, Pôrto KC, Bastos CJP (2011) Checklist de Briófitas da Chapada Diamantina, Bahia, Brasil. Boletim do Instituto de Botânica 21: $111-124$.

Valente EB, Pôrto KC (2006) Hepáticas (Marchantiophyta) de um fragmento de Mata Atlântica na Serra da Jibóia, município de Santa Terezinha, BA, Brasil. Acta Botanica Brasilica 20: 433-441. https://doi.org/10.1590/S0102-33062006000200018

Vieira HCA, Oliveira RR, Silva ML, Silva DL, Conceição GM, Oliveira HC (2017) Briófitas de ocorrência em São João do Sóter, Maranhão, Brasil. Acta Brasiliensis 1: 8-12. https://doi.org/10.22571/ Actabra12201745

Yano O (2011) Catálogo de musgos brasileiros: literatura original, basiônimo, localidade-tipo e distribuição geográfica. Instituto de Botânica, São Paulo, 180 pp.

Yano O, Peralta DF (2011) Flora da Serra do Cipó, Minas Gerais: Briófitas (Anthocerotophyta, Bryophyta e Marchantiophyta. Boletim de Botânica da Universidade de São Paulo 29: 135-211. https://doi. org/10.11606/issn.2316-9052.v29i2p135-299

Zander RH (1993) Genera of the Pottiaceae: mosses of harsh environments. Bulletin of the Buffalo Society of Natural Sciences 32: $1-378$.

Zander RH (1995) Phylogenetic relationships of Hyophiladelphus gen. nov. (Pottiaceae, Musci) and a perspective on the cladistic method. Bryologist 98: 363-374. https://doi.org/10.2307/3243374 